BMJ Open Sport \& Exercise Medicine

\title{
Physical activity and exercise in youth mental health promotion: a scoping review
}

\author{
Michaela Pascoe (1) , ${ }^{1,2}$ Alan P Bailey, ${ }^{3}$ Melinda Craike (1) , ${ }^{1,4}$ Tim Carter, ${ }^{5}$ \\ Rhiannon Patten, ${ }^{1}$ Nigel Stepto, ${ }^{1,6,7,8}$ Alexandra Parker ${ }^{1,3}$
}

To cite: Pascoe M, Bailey AP, Craike M, et al. Physical activity and exercise in youth mental health promotion: a scoping review. BMJ Open Sport \& Exercise Medicine 2020;6:e000677. doi:10.1136/ bmjsem-2019-000677

- Additional material is published online only. To view please visit the journal online (http://dx.doi.org/10.1136/ bmjsem-2019-000677).

Accepted 6 December 2019

Check for updates

C Author(s) (or their employer(s)) 2020. Re-use permitted under CC BY-NC. No commercial re-use. See rights and permissions. Published by BMJ.

For numbered affiliations see end of article.

Correspondence to Dr Michaela Pascoe; michaela.pascoe@vu.edu.au

\section{ABSTRACT}

Background/Aim This scoping review examined the breadth and outcomes of controlled trials testing the effect of physical activity and exercise interventions across all mental health outcomes for mental health promotion and indicated prevention studies in young people.

Methods The literature search was conducted using 'Evidence Finder'.

Results Thirty publications were included. Available evidence suggested that interventions of varying intensity may lead to a reduction in depression symptoms and that moderate-to-vigorous-intensity and light-intensity interventions may reduce anxiety symptoms. Effects of physical activity/exercise interventions on additional mental health outcomes were also shown; however, the number of studies was small, indicating a limited evidence base. Robust research regarding the effects of physical activity/exercise on mental health promotion and as an indicated prevention strategy in young people is lacking. Conclusion The available evidence suggests that physical activity/exercise is a promising mental health promotion and early intervention strategy and warrants further investigation.

\section{INTRODUCTION}

Youth mental health disorders are expected to be a leading cause of disability in developed countries by $2020 .{ }^{1}$ Therefore, the promotion of mental health and the prevention of mental health disorders are paramount. Significantly impaired function is associated with increased mental health disorder symptoms in young people, such as depression and anxiety symptoms, which are equally, if not more, prevalent than diagnosed disorders. ${ }^{2-4}$ Indeed, young people with increased risk of or increased incidence of mental disorder symptoms have significant social and occupational functioning impairments and experience high levels of psychological distress. ${ }^{5}$ Given the high prevalence, interventions that promote mental health as well as target the early phases or subthreshold levels of disorder are urgently required. ${ }^{6}$

\section{What is already known}

- Considerable impairment in functioning is associated with elevated mental disorder symptoms in young people, which are equally, if not more, prevalent than diagnosed disorders.

- Young people are often reluctant to seek help for mental health concerns; therefore, interventions need to be youth-friendly, acceptable, feasible and non-stigmatising.

- Physical activity/exercise is a non-stigmatising intervention with few side effects and is viewed by young people as helpful in promoting mental health and treating mental health problems.

\section{What are the new findings}

Interventions of varying intensity may lead to a reduction in depression symptoms.

- Moderate-to-vigorous-intensity and light-intensity interventions may reduce anxiety symptoms.

- Effects of physical activity/exercise interventions on additional mental health outcomes were also shown however, the number of studies was small, indicating a limited evidence base.

Access to interventions that promote mental health and that are effective in the early stages of illness has the potential to rapidly improve the mental health and functioning of young people and prevent the negative impact of persistent mental illness. ${ }^{7}$ Young people are often reluctant to seek help for mental health concerns; therefore, interventions need to be youth-friendly, acceptable, feasible and nonstigmatising $^{8}$ and matched to their specific needs. ${ }^{9-11}$

Physical activity (PA)/exercise is a nonstigmatising intervention with few side effects ${ }^{12}$ and is viewed by young people as helpful in promoting mental health and treating mental health problems. ${ }^{13}$ Despite these positive beliefs, disengagement from regular exercise, PA and sporting clubs steadily increases during adolescence, ${ }^{14} 15$ 
coinciding with the average age of onset of mental health problems. $^{16}$

\section{Rationale}

While emerging evidence indicates that PA/exercise is effective in reducing depression symptoms in young people, ${ }^{17} 18$ few studies have examined PA/exercise as a mental health promotion strategy for other mental health problems. One single meta-analysis has demonstrated the benefits of PA on mental health in adolescents up to the age of 18 years. ${ }^{19}$ There are no existing systematic or scoping reviews across all youth mental health outcomes of PA/exercise for mental health promotion and indicated prevention that include people aged up to 25 years.

\section{Objectives}

This review addressed the following research question: what is known about the effectiveness of PA/exercise for mental health promotion and as an indicated prevention strategy in young people? (in all contexts: university/ school, community, hospital, correctional facility). 'Physical activity' is defined as any bodily movement produced by skeletal muscles that results in energy expenditure, and 'exercise' is the planned, structured and repetitive undertaking of PA for the purpose of maintaining or improving health-related or skill-related components of physical fitness. ${ }^{2021}$ The term 'mental health outcomes' refers to mental health symptoms collected by specific quantitative outcome measures of symptom severity, for example, depression symptoms as measured using the Depression Anxiety Stress Scale. It also refers to general indicators of mental health, such as quality of life and well-being, ${ }^{22}$ including depression symptoms, anxiety symptoms, stress, mood states, substance use, self-esteem, affect, resilience, self-confidence and self-concept.

We aimed to provide a comprehensive overview of the breadth and outcomes of controlled trials testing the effect of PA/exercise across all mental health outcomes. Therefore we included both mental health promotion and indicated prevention studies. We also aimed to describe the nature of the interventions, including specification of dose of the activity, as an initial contribution to informing translation into practice. We have selected these outcomes so that the findings can be interpreted in relation to current government guidelines for recommended amounts of PA engagement in the general population, which focus on engagement in moderatevigorous-intensity $\mathrm{PA} /$ exercise. $^{23-25}$

The following were the objectives:

- To examine the extent and range of outcomes from $\mathrm{PA} /$ exercise prevention interventions in a youth mental health context.

- To collate mental health, quality-of-life and functioning outcomes.

- To present an overview of the impact of PA/exercise across mental health symptomology.

- To examine the specification of dose (intensity) of PA/exercise interventions.

\section{METHODS}

\section{Protocol and registration}

The review was conducted and reported according to the Preferred Reporting Items for Systematic Reviews and Meta-Analyses extension for scoping reviews (PRISMA-ScR) guidelines ${ }^{26}$ and the five-stage framework outlined in Arksey and O'Malley. ${ }^{27}$ A review protocol was not published.

\section{Eligibility criteria}

To determine study eligibility, the following criteria were applied: young people (mean age between 12 and 25.9 years); published from 1980 to 2017 as youth mental health was not considered a discrete field prior to $1980^{28}$; included a PA/exercise intervention and a comparison condition; reported on the quantitative effects of at least one mental health symptom, such as depression symptoms; PA/exercise interventions lasted longer than 3 weeks in duration ${ }^{29}{ }^{30}$; study designs were randomised controlled trials (RCTs) or non-RCTs; and studies were published in English. Excluded studies were unpublished studies or studies with a population that had a diagnosis of a mental disorder, as we have examined PA/exercise as a treatment for mental illness in our companion paper to this article, ${ }^{31}$ and non-intervention studies.

\section{Information sources}

We have included a broad range of outcomes associated with the risk of mental health problems, such as stress and body image, and examination of the dose of PA/ exercise in the interventions. The search was conducted using 'Evidence Finder' (www.orygen.org.au), which is a comprehensive database of all available published controlled trials and systematic reviews of interventions in the youth mental health field. ${ }^{28}{ }^{32}$ The 'Evidence Finder' is an Australian initiative developed by Orygen, The National Centre of Excellence in Youth Mental Health and headspace, National Youth Mental Health Foundation. ${ }^{26} 27$ The searchable database is populated annually using comprehensive and systematic searches of the Embase, MEDLINE, PsycINFO and Cochrane Library databases, coupled with strict and reproducible inclusion criteria to identify studies (see refs 2627 for detailed methodology). It includes research published from 1980 to 2017 and contains all available prevention, treatment and relapse prevention studies in young people (mean age 6-25 years), across the following mental illnesses: anxiety, depression, bipolar, eating disorders, psychosis, substance use and suicide-self harm. It contains controlled trials (including RCTs and quasi-randomised studies), systematic reviews and meta-analyses, published in English. Unpublished trials are not included within the Evidence Finder. Searches were conducted in July 2018 (updated April 2019) and all studies classified as 'Physical activity/Exercise' were screened. Reference lists of identified literature were searched for suitable primary research based on titles in the first instance, and if relevant abstracts and full-text review. 


\section{Search}

The following criteria were applied to the 'Evidence Finder' search engine (https://www.orygen.org.au/ Training/Evidence-Finder): (1) mental health or substance use problem: 'all'; (2) stage of illness: 'all'; (3) treatment/intervention: 'complementary and alternative interventions (CAM)', followed by 'Physical activity/exercise'; (4) publication date: 'all'; and (5) keyword: none. Advanced options selected include systematic reviews, RCTs and controlled clinical trials.

\section{Selection of sources of evidence}

Title/abstract screening was undertaken by two authors (MP, AP). Full texts were independently reviewed by two authors (MP, AP, MC). There were no conflicts.

\section{Data charting process}

Data charting $^{27}$ was undertaken by a single author (MP) using a specifically designed extraction form (online supplementary tables 1 and 2). Data charting was done independently by a single author and extraction was checked by a second author (TC, APB). Data were obtained directly and only from the published articles.

Two assessors (NS, RP) reviewed each study for objective (heart rate (HR), \% maximal $\mathrm{HR}$, \% $\mathrm{HR}$ reserve, $\% 1-$ repetition maximum, per cent of maximal oxygen uptake $\left.\left(\% \mathrm{VO}_{2 \max }\right)\right)$ and subjective (ratings of perceived exertion) measures of PA/exercise intensity. Using these measures, interventions were classified as light intensity, light to moderate intensity, moderate intensity, moderate to vigorous intensity, or vigorous intensity for aerobic ${ }^{30}$ and resistance exercise. ${ }^{33}$ In cases where the exercise interventions were poorly described, we attempted, where possible, to estimate an exercise intensity based on the compendium of exercise energy expenditure. ${ }^{34} \mathrm{We}$ did not contact authors when insufficient information was provided in the published articles.

\section{Data items}

The following data were extracted: mental health outcomes assessed, tools used to measure mental health outcomes assessed, country of study origin, setting of study conduct, study design type, participants, sample size, mean age, overall findings, assessment timepoints if intention-to-treat analysis was used (as shown in online supplementary table 1), characteristics of the intervention, characteristics of the control group, personnel delivering the intervention, delivery format, and duration and frequency of the delivered intervention (as shown in online supplementary table 2).

\section{Critical appraisal of individual sources of evidence}

A critical appraisal of individual sources of evidence was not conducted in the current scoping review.

\section{Synthesis of results}

A 'descriptive-analytical' method was applied which involved applying a common analytical framework, which was the mental health outcome. ${ }^{27}$

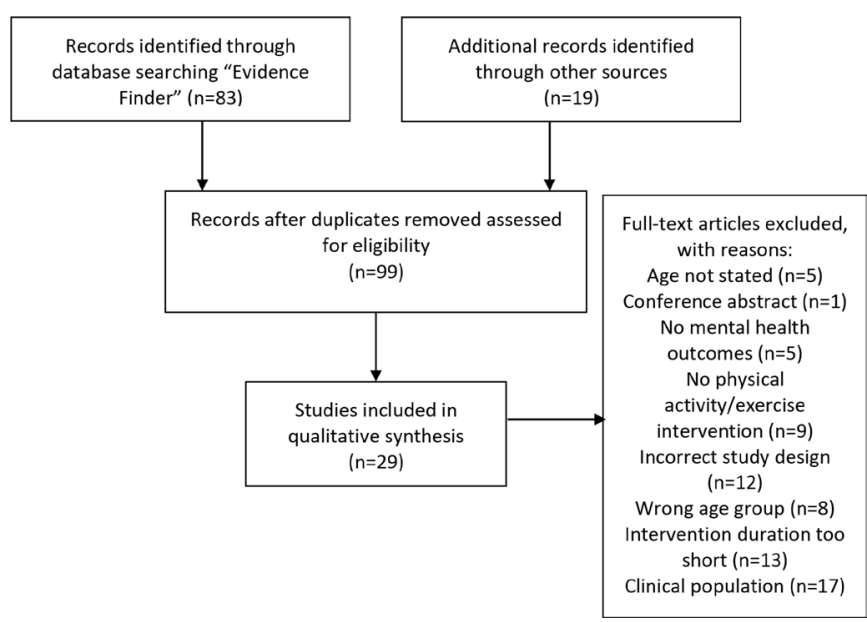

Figure 1 PRISMA flow diagram showing reasons for study exclusions. PRISMA, Preferred Reporting Items for Systematic Reviews and Meta-Analyses.

$\mathrm{PA} /$ exercise interventions generally vary the dose, where the combination of intensity (light, moderate or vigorous) and duration ( $\mathrm{min} /$ week) determines the session and intervention dose. Due to heterogeneity of the duration of interventions and that PA/exercise intensity is strongly linked to affective responses and sustainability, we focused on PA/exercise intervention intensity.

\section{RESULTS}

\section{Selection of sources of evidence}

Eighty-three records were returned in 'Evidence Finder' and were screened for eligibility, and an additional 19 eligible papers were identified via screening of the references lists of reviews identified by 'Evidence Finder'. As shown in figure 1, after full-text screening, a total of 29 studies met the inclusion criteria and were included in the current scoping review.

\section{Characteristics of sources of evidence}

Online supplementary table 1 shows that 4 trials were not randomised, ${ }^{35-38} 1$ was a cross-over RCT, ${ }^{39} 5$ were cluster RCTs $^{40-44}$ and 20 were RCTs. Eight studies included young people with elevated mental disorder symptoms at baseline, including anxiety sensitivity, ${ }^{45}$ anxiety or depression symptoms, ${ }^{37-39} 4647$ or high substance use, ${ }^{48} 49$ and the remaining 22 involved young people without mental health symptoms. Figure 2 shows the distribution of mental health outcomes studied. Online supplementary table 2 shows that 3 interventions were delivered individually, 9 did not state the mode of delivery and 18 were group-based. All but seven studies delivered supervised $\mathrm{PA} /$ exercise interventions. These seven studies did not specify if interventions were supervised or not.

\section{Results of sources of evidence}

The relevant extracted data relating to the review questions and objectives are shown in online supplementary tables 1 and 2. 


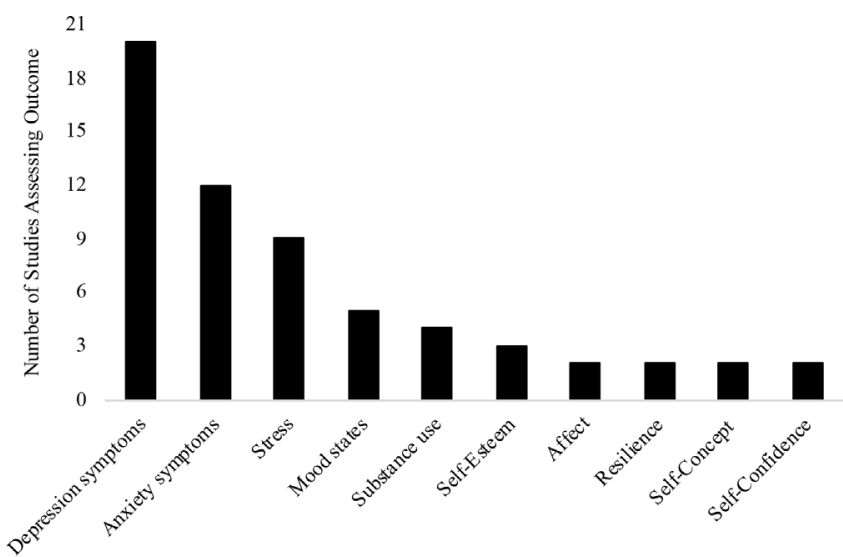

Figure 2 Distribution of mental health outcomes studied. The following outcomes were measured only once and are not depicted in figure 1: anger, anxiety sensitivity, body image, burn-out, fatigue, functioning, hopefulness, life purpose and satisfaction, mindfulness, psychological symptoms and distress, quality of life, self-perception, situational motivation, and social skills.

\section{Types of interventions}

Figure 3 shows the distribution of interventions studied by $\mathrm{PA} /$ exercise intensity, most commonly delivered in supervised group settings. Moderate-to-vigorous-intensity interventions were the most frequently examined, encompassing a broad range of activities, including strength training, gymnastics, enhanced physical education classes and aerobic exercises. Moderate-intensity interventions formed a considerable portion of those examined, and included activities such as team sports, cycling, jogging and exercising to music. Finally, a smaller proportion of studies examined light-intensity activities, including yoga, t'ai chi, and conditioning or stretching exercises.

\section{Synthesis of results}

\section{Depression symptoms}

Twenty studies assessed depression symptoms. As several studies included more than one active intervention condition (ie, three-arm or four-arm studies), the number of comparisons reported here is greater than 20. Three studies assessed vigorous-intensity interventions, and

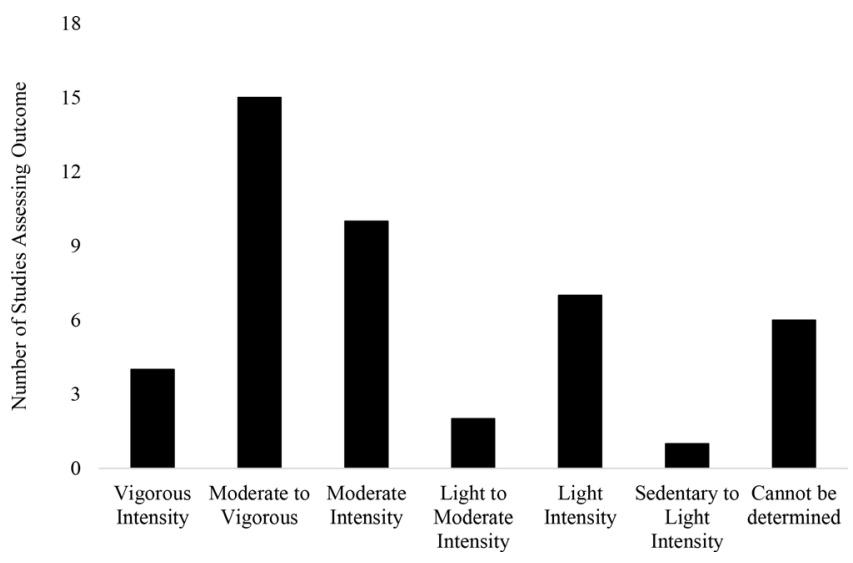

Figure 3 Distribution of interventions studied by intensity. two of these showed decreases in depression symptoms. One of these included incarcerated male adolescents and compared a vigorous-intensity with a moderateintensity intervention. ${ }^{35}$ The second included university students and compared vigorous-intensity $\mathrm{PA} /$ exercise with progressive muscle relaxation (PMR); however, in this study, a reduction in depression symptoms was only reported at mid-intervention. ${ }^{50}$ In the third study, a vigorous-intensity intervention did not improve depression symptoms in unemployed young men, compared with a wait-list group. ${ }^{51}$

Three studies assessed moderate-to-vigorous-intensity interventions and a further three assessed interventions of unspecified intensity, but likely moderate-to-vigorous. In five of these, the interventions decreased depression symptoms. ${ }^{47} 52-55$ Four of these compared moderateto-vigorous-intensity with no intervention, ${ }^{47}$ 53-55 and the remaining study used a standard rehabilitation programme. $^{52}$ The sixth study compared two moderateto-vigorous-intensity interventions and found no effect of either on depression symptoms. ${ }^{40}$

Three studies delivered moderate-intensity interventions and a further four assessed interventions of unspecified intensity, but likely moderate. In two of these, moderate-intensity interventions decreased depression symptoms, one compared with a wait-list group ${ }^{39}$ and the other with health promotion, but only in the subsample of adolescents with elevated baseline depression symptoms. ${ }^{42}$ In the remaining five studies, moderate-intensity interventions had no effect on depression symptoms, compared with wait-list, ${ }^{56}$ no intervention, ${ }^{37}{ }^{57}$ vigorousintensity intervention ${ }^{35}$ or health education. ${ }^{41}$

One study assessed a light-moderate-intensity intervention and found no reduction in depression symptoms in women with high anxiety sensitivity, compared with health education. ${ }^{45}$ One study assessed a light-intensity intervention ${ }^{56}$ and a further three assessed interventions of unspecified intensity, but likely light-intensity. ${ }^{38558} \mathrm{In}$ one of these, the light-intensity intervention decreased depression symptoms compared with no intervention. ${ }^{38}$ The remaining three studies found no effect. ${ }^{555658} \mathrm{~A}$ single study assessed a psychological intervention with a light-intensity component, finding it decreased depression symptoms in university students, compared with a moderate-intensity intervention and no intervention. ${ }^{57}$

Two studies did not provide sufficient information to assess intervention intensity, and neither showed reductions in depression symptoms. ${ }^{36} 46$

\section{Anxiety symptoms}

Twelve studies assessed anxiety symptoms, and six studies included more than one active intervention condition. In five studies, PA/exercise reduced anxiety symptoms. ${ }^{3840525759}$ One study assessed a vigorous-intensity intervention and found no effect in university students with a high number of negative life events, compared with PMR or no intervention. ${ }^{50}$ One study assessed a moderateto-vigorous-intensity intervention and found that both it 
and a likely light-intensity intervention decreased anxiety in university students, with no difference between the groups. ${ }^{55}$ A further five studies did not specify intensity but were likely moderate-to-vigorous. ${ }^{40} 47525960$ In two of these, the moderate-to-vigorous-intensity intervention reduced anxiety symptoms in adolescents compared with a second moderate-to-vigorous intervention (two types of physical education classes) ${ }^{40}$ and in adolescents in juvenile detention, compared with a rehabilitation programme. ${ }^{52}$ An additional study analysed the results of two moderate-to-vigorous-intensity interventions, by combining the resistance and aerobic exercise groups together, and found that these reduced anxiety in male university students with elevated anxiety/depression symptoms, compared with no intervention. ${ }^{47}$ The fourth study found that a moderate-to-vigorous-intensity intervention reduced anxiety symptoms from preintervention to postintervention in university students. This study however did not state whether the moderate-to-vigorousintensity intervention influenced anxiety symptoms compared with the no-intervention group. ${ }^{60}$ The fifth study found a moderate-to-vigorous-intensity intervention had no effect in university students, compared with a stress management intervention or no intervention. ${ }^{59}$

Four studies did not specify intensity, but were likely moderate. In two of these, moderate-intensity interventions did not reduce anxiety compared with health promotion education. ${ }^{41} 42$ In another study, both a likely moderate-intensity intervention and a stress management intervention decreased anxiety, but a likely moderate-tovigorous-intensity intervention did not. ${ }^{59}$ In the fourth study, a moderate-intensity intervention did not reduce anxiety, but a light-intensity intervention that also contained mindfulness and cognitive components did reduce anxiety, compared with no intervention. ${ }^{57}$

One study found a light-to-moderate-intensity intervention did not reduce anxiety symptoms in women with high anxiety sensitivity, compared with health education. ${ }^{45}$ Three studies did not specify intensity but were likely light intensity, and all decreased anxiety in university students. ${ }^{38557}$ In one of these, a second lightintensity intervention did not improve anxiety compared with no intervention. ${ }^{38}$ The remaining two studies, as described above, found that the light-intensity interventions reduced anxiety as effectively, ${ }^{55}$ or more effectively, than moderate-intensity interventions. ${ }^{57}$

\section{Stress}

Nine studies assessed stress. As several studies included more than one active intervention condition, the number of comparisons reported here is greater than nine. Two studies delivered a vigorous-intensity intervention, and one of these found it decreased stress in adolescents, compared with no intervention. ${ }^{61}$ The second study found that the intervention did not decrease stress among unemployed young men, compared with a waitlist group. ${ }^{51}$
Three studies assessed a moderate-to-vigorous-intensity intervention or a likely moderate-to-vigorous-intensity intervention. ${ }^{44} 53$ In one of these, the intervention decreased stress, as did a moderate-intensity intervention and a psychological intervention, compared with no intervention, in university students. ${ }^{59}$ In the remaining two studies moderate-to-vigorous-intensity intervention had no effect on stress in individuals who were included on the basis of low levels of PA, compared with no intervention, ${ }^{53}$ or in adolescents when compared with a light-intensity intervention. ${ }^{44}$

One study of a moderate-intensity intervention found that it decreased stress in adolescents, compared with no intervention, ${ }^{61}$ and a further three did not specify intervention intensity but were likely moderate. ${ }^{43} 5759$ In one of these, both the moderate-intensity intervention and the psychological treatment condition decreased stress in university students, compared with no intervention, as stated above. ${ }^{59}$ In the remaining two studies ${ }^{43} 57$ moderate-intensity interventions had no effect on stress compared with a likely light-intensity intervention ${ }^{43}$ or no intervention. ${ }^{57}$

One study assessed a light-to-moderate-intensity intervention and found that it decreased stress in women with high anxiety sensitivity, compared with health education. ${ }^{45}$ Three studies did not specify intervention intensity but were likely light-intensity. ${ }^{38} 4344$ Only one of these found that the intervention decreased stress in university students with elevated combined symptoms of depression, anxiety and stress, compared with no intervention, ${ }^{38}$ with the remaining two interventions showing no effect on stress compared with a moderate-intensity intervention ${ }^{43}$ and a moderate-to-vigorous-intensity intervention $^{44}$

\section{Mood states}

Five studies assessed mood states. One study assessed a vigorous-intensity intervention and found it improved mood states in adolescents, compared with a moderateintensity intervention and no intervention. ${ }^{61}$

Two studies examined moderate-to-vigorous-intensity interventions, ${ }^{61}{ }^{62}$ and a further two did not specify intensity but were likely moderate-to-vigorous-intensity. ${ }^{44} 52 \mathrm{In}$ two of these, the moderate-to-vigorous-intensity interventions improved mood states, compared with a rehabilitation programme ${ }^{52}$ and no intervention. ${ }^{62}$ In one of these, the comparison interventions (PMR and discussion) also improved mood states (stress only) compared with no intervention. ${ }^{62}$ Moderate-to-vigorousintensity intervention had no effect on mood states in the remaining two studies, compared with no intervention ${ }^{61}$ or a light-intensity intervention. ${ }^{44}$

Two studies examined moderate-intensity interventions, and neither had an effect on mood states in adolescents, compared with no intervention ${ }^{61}$ or a lightintensity intervention. ${ }^{43}$ The second study found that the light-intensity intervention improved mood states. ${ }^{43}$ 
Table 1 Percentage (frequency) of total reviewed studies that showed beneficial effects of physical activity (PA)/exercise interventions compared with a control intervention

\begin{tabular}{|c|c|c|c|c|}
\hline Comparison group & Depression symptoms & Anxiety symptoms & Stress & Mood states \\
\hline $\begin{array}{l}\text { Compared with a non- } \\
\text { PA/exercise control group }\end{array}$ & $50 \%(n=3 \text { out of } 6)^{424652}$ & $16 \%(n=1 \text { out of } 6)^{52}$ & $33 \%(n=1 \text { out of } 3)^{61}$ & $100 \%(n=3 \text { out of } 3)^{526183}$ \\
\hline $\begin{array}{l}\text { Compared with a wait-list } \\
\text { or no intervention }\end{array}$ & $\begin{array}{l}45 \%(n=5 \text { out of } 11)^{38} 39 \\
535457\end{array}$ & $\begin{array}{l}66 \%(n=4 \text { out of } 6)^{38} \\
475759\end{array}$ & $\begin{array}{l}66 \%(4 \text { out of } 6)^{3845} \\
5761\end{array}$ & $50 \%(n=1 \text { out of } 2)^{83}$ \\
\hline $\begin{array}{l}\text { Compared with a second } \\
\text { PA/exercise intervention }\end{array}$ & $66.6 \%(n=2 \text { out of } 3)^{3555}$ & $50 \%(n=1 \text { out of } 2)^{40}$ & $0 \%(n=0$ out of 2$)$ & $100 \%(n=2 \text { out of } 2)^{4344}$ \\
\hline
\end{tabular}

\section{Summary of mood state findings}

Table 1 shows the percentage and frequency of the total number of reviewed studies that showed beneficial effects of a PA/exercise intervention compared with (1) a non-PA/exercise control group intervention, (2) a wait-list or no intervention and (3) a second PA/ exercise intervention. Table 2 shows the percentage and frequency of the total number of reviewed studies that showed beneficial effects of a PA/exercise according to the intensity of the delivered intervention.

\section{Substance use}

Four studies measured substance use. In one of these, a vigorous-intensity intervention decreased methamphetamine use in unemployed young men, compared with a wait-list group. ${ }^{51}$ In another, the likely moderateintensity intervention decreased substance use, when delivered to university students with high anxiety sensitivity scores, compared with health education. ${ }^{42}$ The remaining two studies compared two PA/exercise interventions in sedentary university students who engaged in hazardous drinking. In one of these, contracting to complete light-moderate-intensity activities plus contingency management did not influence substance use, compared with a single session of motivational enhancement therapy for exercise. ${ }^{48}$ These same authors later found that both contingency management (to contract to complete moderate-to-vigorous activities) and contingency management, in addition to reinforcing participants for completion of moderate-to-vigorous exercise activities, decreased alcohol use, with no difference between the groups. ${ }^{49}$
Self-esteem

Three studies measured self-esteem. One study found that a moderate-to-vigorous-intensity intervention improved self-esteem in adolescents, compared with a second moderate-to-vigorous-intensity intervention (two variations of physical education classes) ${ }^{40} \mathrm{~A}$ second study found that a moderate-to-vigorous-intensity intervention improved self-esteem compared with a rehabilitation programme in adolescents in juvenile detention. ${ }^{52}$ The third study found that an unspecified, likely light-intensity intervention improved self-esteem in university students and employees, compared with no intervention. ${ }^{58}$

\section{Affect}

Two studies measured affect and both included a moderate-intensity and light-intensity intervention, or unspecified but likely moderate-intensity and lightintensity. In one study, neither intervention influenced affect compared with wait-list in children with obesity. ${ }^{56}$ In the second study, the light-intensity intervention improved affect compared with the moderate-intensity intervention in adolescents. ${ }^{43}$

\section{Resilience}

Two studies measured resilience and compared two PA/ exercise interventions in adolescents. In one of these the light-intensity intervention increased resilience while the moderate-to-vigorous-intensity intervention decreased resilience. ${ }^{44}$ In the second study, there was no difference between a light-intensity intervention and a moderateintensity intervention. ${ }^{43}$

Table 2 Percentage (frequency) of total reviewed studies that showed beneficial effects of physical activity/exercise interventions depending on the intensity of the intervention

\begin{tabular}{|c|c|c|c|c|}
\hline Intensity of the intervention & Depression symptoms & Anxiety symptoms & Stress & Mood states \\
\hline Vigorous-intensity intervention & $33 \%(n=1 \text { out of } 3)^{35}$ & $0 \%(n=0$ out of 1$)$ & $50 \%(n=1 \text { out of } 2)^{43}$ & $100 \%(n=1 \text { out of } 1)^{61}$ \\
\hline $\begin{array}{l}\text { Moderate-to-vigorous-intensity } \\
\text { intervention }\end{array}$ & $\begin{array}{l}83 \%(n=5 \text { out of } 6)^{47} \\
52-55\end{array}$ & $\begin{array}{l}66 \%(n=4 \text { out of } 6)^{40} \\
475255\end{array}$ & $33 \%(n=1 \text { out of } 3)^{59}$ & $66 \%(n=2 \text { out of } 3)^{5283}$ \\
\hline Moderate-intensity intervention & $28 \%(n=2 \text { out of } 7)^{3942}$ & $25 \%(n=1 \text { out of } 4)^{59}$ & $33 \%(n=1 \text { out of } 3)^{59}$ & $0 \%(n=0$ out of 2$)$ \\
\hline Light-moderate-intensity intervention & $0 \%(n=0$ out of 1$)$ & None identified & $\begin{array}{l}100 \%(n=1 \text { out of } \\
1)^{45}\end{array}$ & None identified \\
\hline Light-intensity intervention & $25 \%(n=1 \text { out of } 4)^{38}$ & $\begin{array}{l}75 \%(n=3 \text { out of } 4)^{38} \\
5557\end{array}$ & $33 \%(n=1 \text { out of } 3)^{38}$ & $0 \%(n=0$ out of 2$)$ \\
\hline
\end{tabular}




\section{Self-concept}

Two studies measured self-concept, which refers to one's views, attitudes and beliefs about the self. In one, a vigorous-intensity intervention improved self-concept, compared with a likely moderate-intensity intervention, in incarcerated adolescents. ${ }^{35}$ In the second study, a lightintensity intervention improved self-concept, compared with a likely moderate-to-vigorous-intensity intervention in secondary schools students. ${ }^{63}$

\section{Self-confidence}

Two studies measured self-confidence. A moderate-tovigorous-intensity intervention improved self-confidence compared with no intervention in university students. ${ }^{60}$ There was no difference between a likely light-intensity intervention and moderate-intensity intervention, both of which did not influence self-confidence during periods of stressful life events in secondary school students. ${ }^{44}$

\section{Outcomes reported once}

Both a light-to-moderate-intensity intervention and a health education intervention reduced anxiety sensitivity in women with high anxiety sensitivity, with no difference between groups. ${ }^{45}$ A likely light-intensity intervention improved fatigue and quality of life, while a resistancebased intervention of unspecified intensity improved body image, in university students and employees, compared with no intervention. ${ }^{58}$ Hopefulness increased following two likely light-intensity interventions in university students with elevated depression symptoms compared with no intervention. ${ }^{38}$ A likely light-intensity intervention and a moderate-intensity intervention had no effect on mindfulness, anger expression and positive psychology, ${ }^{43}$ or on life purpose and satisfaction, in secondary school students. ${ }^{44}$ The likely light-intensity intervention did, however, improve anger control. ${ }^{44}$ Light-intensity intervention or moderate-intensity intervention had no effect on scholastic competence and social competence in children with obesity; however, in this same study, the moderate-intensity intervention improved physical self-worth, global self-worth, strength competence and attractive body adequacy, and the light-intensity intervention improved global self-worth, compared with wait-list. ${ }^{56}$ Social skills improved following a likely moderate-intensity intervention in university students with high anxiety sensitivity, compared with health education. ${ }^{42}$ A likely moderate-intensity intervention, a likely moderate-to-vigorous-intensity intervention or stress management did not decrease personal burn-out among university students compared with no intervention. ${ }^{59}$ Vigorous-intensity intervention had no effect on psychological symptoms and distress in university students, compared with no intervention or PMR. ${ }^{50}$ Situational motivation increased following an intervention of unspecified intensity in university students, compared with stretching. ${ }^{46}$

\section{Intensity of interventions}

Of the studies that examined vigorous-intensity interventions, a considerable proportion found positive outcomes on depression symptoms, ${ }^{35} 50 \quad 51$ stress $^{43}$ and mood states, ${ }^{61}$ but not for anxiety. ${ }^{50}$ Moderateto-vigorous-intensity interventions showed improvements in depression symptoms, ${ }^{40} 4752-55$ anxiety symptoms, ${ }^{40} 4752555960$ self-esteem ${ }^{40} 52$ and mood states. ${ }^{52} 62$ There was less support for moderate-intensity interventions in reducing depression symptoms, with only two positive studies from seven ${ }^{39} 42$ and only one positive study for anxiety ${ }^{59}$ and stress ${ }^{59}$ from the four that measured these outcomes. There was no support for moderate-intensity interventions in improving mood states from the two studies that measured this. The current review identified seven studies assessing the impact of light-intensity interventions on mental health outcomes, and the results of these were generally positive for anxiety, ${ }^{38} 5557$ mood states ${ }^{43} 44$ and affect, ${ }^{43}$ but not for depression ${ }^{555658}$ and stress. ${ }^{38}$

\section{DISCUSSION}

This scoping review examined the extent and range of outcomes from PA/exercise interventions in youth mental health. We presented an overview of the effects of $\mathrm{PA} /$ exercise interventions across mental health outcomes and mental health-related factors. We examined outcomes according to intervention intensity in order to facilitate the translation of research findings into clinical practice.

\section{Summary of evidence}

\section{Elevated mental disorder symptoms}

The range of mental health and quality-of-life outcomes examined may indicate the broad applicability of PA/ exercise as an intervention for emerging mental health problems in young people. Of the eight included studies that recruited participants on the basis of elevated symptoms of mental health disorder at baseline, five demonstrated the effect of $\mathrm{PA} /$ exercise interventions in reducing symptoms of depression ${ }^{38} 394647$ and high substance use, ${ }^{49}$ indicating the potential of PA/exercise as an indicated or targeted prevention strategy.

\section{A mental health-promoting strategy}

Of the 22 included studies that did not recruit participants on the basis of elevated symptoms of mental health disorder, there was a demonstrated effect of $\mathrm{PA} /$ exercise improving mental health in 17 of these: affect, ${ }^{43}$ anxiety symptoms, ${ }^{40} 525759$ body image ${ }^{58}$ depression symptoms, ${ }^{35} 52-5557$ fatigue, ${ }^{58}$ functioning, ${ }^{44}$ mood states, ${ }^{4344526162}$ self-perception, ${ }^{56}$ quality of life, ${ }^{58}$ resilience, ${ }^{44}$ self-concept, ${ }^{35}{ }^{63}$ self-esteem, ${ }^{40}{ }^{52}$ social skills, ${ }^{42}$ stress $^{575961}$ and substance use, ${ }^{42} 51$ indicating that PA/ exercise has a potentially promising effect as a mental health-promoting strategy. 


\section{Future research and practice}

There are indications that outcomes may vary depending on intervention intensity. Given the mixed findings in regard to intensity, a future research agenda could be testing and comparing the feasibility, acceptability and effectiveness of different intensities head to head, to seek a threshold dose for benefit. Current government guidelines for recommended amounts of PA engagement in the general population focus on moderate-vigorousintensity $\mathrm{PA} /$ exercise $^{23-25}$; however, the results of this scoping review indicate that light-intensity PA/exercise shows benefit for some mental health outcomes. This is important as the experience of negative affect when participating in higher-intensity $\mathrm{PA} /$ exercise is more likely to occur compared with participating in lower-intensity PA/exercise. ${ }^{64}$ As affect appears to be an important factor in predicting adherence to PA/exercise, ${ }^{6465}$ light-intensity interventions may be beneficial for people who are unlikely to adhere to more vigorousintensity interventions or are commencing from a very low baseline level of PA. Light-intensity interventions may also have the capacity to be delivered by a range of professionals across different settings, ${ }^{66}$ be less reliant on group-based formats and may also be a good basis on which to develop self-efficacy for PA/exercise, ${ }^{67}$ which might then lead to uptake of more intensive types of PA/ exercise, ${ }^{68}$ providing impetus to examine these interventions. Given that our review identified findings to indicate that likely light-intensity interventions improve anxiety symptoms ${ }^{3855} 57$ and mood states, ${ }^{43} 44$ further research should be conducted to better understand the potential benefits of light-intensity interventions in youth mental health.

Future research should also consider the importance of self-selection of activity type and intensity as autonomy is proposed as one of the three basic psychological needs fundamental to positive mental health, ${ }^{69} 70$ and as when intensity is self-selected, rather than imposed, participants experience greater tolerance to higher-intensity PA/exercise. ${ }^{64}$ This indicates health promotion strategies should be designed to suit a diverse range of needs and preferences, and should be designed to be driven by individuals, in order to improve uptake and adherence.

The results of the present scoping review highlight the importance of maintaining and building PA and exercise programme in primary, secondary and tertiary education settings in order to promote mental health among young people. In general, preventive interventions are not currently widely implemented in schools and communities. ${ }^{71} 72$ Mendelson et $a l^{73}$ recently highlighted that school-based interventions have small but significant effects in terms of preventing the onset of depression in young people. Even if modestly effective, the populationlevel implementation of prevention interventions would help young people to develop healthy coping strategies that could protect them against the development of depression. ${ }^{74}$ Therefore, it would be valuable in future research to explore the implementation barriers and facilitators to the successful implementation and maintenance of PA programmes in education settings.

\section{Strengths and limitations}

The strengths of this scoping review are that it (1) is the first examining the effects of PA/exercise across mental health outcomes for non-clinical populations of youth aged $12-25$; (2) provides a synthesis of intervention intensity; (3) was conducted in concordance with a framework and reported per PRISMA guidance; and (4) identified the distribution of the evidence base for PA/ exercise as a preventative intervention for mental health, highlighting patterns of positive findings and where more research is needed. A number of limitations exist. Only five studies included follow-up assessments 3942465056 and none assessed whether PA/exercise prevented the onset of future episodes of threshold mental disorder, rendering longer-term preventive effects unknown. As only nine studies included a comparison group that delivered an intervention 414245465052596175 - the remaining included a wait-list or additional PA/exercise group-in the majority of studies it remains unknown if the observed effects are due to PA/exercise or from non-specific time/ attention effects. Only 12 studies provided sufficient information to confidently assess intervention intensity, highlighting a need for better specification and clearer reporting of $\mathrm{PA} /$ exercise interventions. We described interventions in terms of intensity; however, there may be other potentially important aspects such as duration and adherence. Indeed, previous research has demonstrated that the frequency, intensity, time, type and context of PA are all critical factors in mental health outcomes in young people. ${ }^{76}$ These authors highlight that PA provides an opportunity to increase social interaction, self-efficacy and perceived competence, improvements in body image and self-perceptions, as well as autonomy, factors which are commonly agreed to contribute to mental well-being. ${ }^{77} 78$ Indeed in the current scoping review we have not explored the impact of these factors. In the current study we have reviewed the impact of PA/exercise in different populations; however, it is also possible that there may be differences between populations, such as that effect may differ between university students and incarcerated young men. This question is beyond the scope of the current study; however, it is an important area of future research in order to better understand for whom certain interventions are more appropriate. We have not undertaken quality appraisal of individual trials, given it is outside the scope of this review. In future studies, it might also be worth searching a wider range of databases than those covered by 'Evidence Finder', as well as grey literature using ProQuest dissertations and theses.

\section{CONCLUSIONS}

This review aimed to determine the breadth and outcomes of intervention studies testing the effect of PA/exercise across mental health outcomes in young 
people who may or may not have been currently experiencing elevated symptoms of mental health disorders. We found evidence that PA/exercise interventions of varying intensities may decrease depression symptoms and some evidence that moderate-to-vigorous-intensity and lightintensity interventions reduce anxiety symptoms.

The mood-enhancing effects of light-intensity interventions have not been as widely researched, but the limited available evidence is positive, highlighting the need to explore the effectiveness and maintenance of PA interventions across the intensity spectrum. ${ }^{79}$ In adults, mental health benefits are associated with lower levels of PA than required for physical health benefits, ${ }^{80-82}$ and it is possible that this relationship also holds for younger people; however, this would require additional rigorous empirical support.

Investigation of the effects of PA/ exercise interventions using robust designs is a research priority. Significant effects of PA/exercise interventions on additional mental health outcomes were identified in this review; however, few studies were available and findings were inconsistent, indicating that research regarding the impact of PA/ exercise interventions on mental health outcomes, other than depression, is lacking.

This review found preliminary evidence for the beneficial effects of PA/exercise interventions as a mental health promotion and early intervention strategy among young people, and future research is required to confirm or refute these findings and fill identified gaps in knowledge. We recommend that future studies include follow-up assessments and appropriate control groups not engaging in PA/exercise. Studies should specify the details of the PA/exercise interventions supported with quantifiable measures, to facilitate replicability and translation into practice. Findings from this review will be valuable for the design of future PA/exercise intervention studies attempting to prevent or intervene early with mental health problems commonly emerging in adolescence and early adulthood.

\section{Author affiliations \\ ${ }^{1}$ Institute for Health and Sport, Victoria University, Melbourne, Victoria, Australia ${ }^{2}$ Cancer Experiances Reasearch, Peter MacCallum Cancer Centre, Melbourne, Victoria, Australia \\ ${ }^{3}$ Orygen, The National Centre of Excellence in Youth Mental Health and Centre for Youth Mental Health, University of Melbourne, Parkville, Victoria, Australia ${ }^{4}$ Mitchell Institute, Victoria University, Melbourne, Victoria, Australia ${ }^{5}$ Institute of Mental Health, School of Health Sciences, University of Nottingham, Nottingham, UK \\ ${ }^{6}$ Monash Centre for Health Research and Implementation, Monash University, Melbourne, Victoria, Australia \\ ${ }^{7}$ Australian Institute for Musculoskeletal Science, Victoria Universit, Melbourne, Victoria, Australia \\ ${ }^{8}$ Medicine-Western Health, Faculty of Medicine, Dentistry and Health Science, Melbourne University, Melbourne, Victoria, Australia}

Contributors MP, AP, MC conducted the literature search. MP designed the figures and tables. All authors contributed to study design. MP, AP, APB, NS, RP, TC contributed to data collection. MP, AP, APB, NS, RP, TC contributed to data analysis and data interpretation. All authors contributed to writing and reviewing the manuscript.
Funding The authors have not declared a specific grant for this research from any funding agency in the public, commercial or not-for-profit sectors.

Competing interests None declared.

Patient consent for publication Not required.

Provenance and peer review Not commissioned; externally peer reviewed.

Open access This is an open access article distributed in accordance with the Creative Commons Attribution Non Commercial (CC BY-NC 4.0) license, which permits others to distribute, remix, adapt, build upon this work non-commercially, and license their derivative works on different terms, provided the original work is properly cited, appropriate credit is given, any changes made indicated, and the use is non-commercial. See: http://creativecommons.org/licenses/by-nc/4.0/.

\section{ORCID iDs}

Michaela Pascoe http://orcid.org/0000-0002-3831-5660

Melinda Craike http://orcid.org/0000-0002-7374-1286

\section{REFERENCES}

1 WHO. Global health risks: mortality and burden of disease attributable to selected major risks. Geneva: World Health Organisation, 2009.

2 Judd LL, Akiskal HS, Paulus MP. The role and clinical significance of subsyndromal depressive symptoms (SSD) in unipolar major depressive disorder. J Affect Disord 1997;45:5-18. discussion -8.

3 Judd LL, Paulus MP, Wells KB, et al. Socioeconomic burden of subsyndromal depressive symptoms and major depression in a sample of the general population. Am J Psychiatry 1996;153:1411-7.

4 Rivas-Vazquez RA, Saffa-Biller D, Ruiz I, et al. Current issues in anxiety and depression: comorbid, mixed, and subthreshold disorders. Prof Psychol 2004;35:74-83.

5 Scott EM, Hermens DF, Glozier N, et al. Targeted primary carebased mental health services for young Australians. Med J Aust 2012;196:136-40.

6 McGorry PD, Hickie IB, Yung AR, et al. Clinical staging of psychiatric disorders: a heuristic framework for choosing earlier, safer and more effective interventions. Aust N Z J Psychiatry 2006;40:616-22.

7 Hetrick SE, Parker AG, Hickie IB, et al. Early identification and intervention in depressive disorders: towards a clinical staging model. Psychother Psychosom 2008;77:263-70.

8 Rickwood D, Deane FP, Wilson CJ, et al. Young people's helpseeking for mental health problems. Australian e-Journal for the Advancement of Mental Health 2005;4:218-51.

9 Rickwood DJ, Deane FP, Wilson CJ. When and how do young people seek professional help for mental health problems? Med $\mathrm{J}$ Aust 2007;187:S35-9.

10 Wyn J. The transition of young people to adulthood in changing times and contexts. In: Galbally R, ed. Healthy minds, healthy bodies, healthy nation: connecting education and health. Canberra: Australian College of Education, 2004a.

11 Wyn J. Becoming adult in the 2000s: new transitions and new careers. Family Matters 2004;68:4-10.

12 Chu I-H, Buckworth J, Kirby TE, et al. Effect of exercise intensity on depressive symptoms in women. Ment Health Phys Act 2009;2:37-43.

13 Jorm AF, Wright A. Beliefs of young people and their parents about the effectiveness of interventions for mental disorders. Aust N Z J Psychiatry 2007;41:656-66.

14 Zimmermann-Sloutskis D, Wanner M, Zimmermann E, et al. Physical activity levels and determinants of change in young adults: a longitudinal panel study. Int J Behav Nutr Phys Act 2010;7:2-13.

15 Eime RM, Harvey JT, Sawyer NA, et al. Changes in sport and physical activity participation for adolescent females: a longitudinal study. BMC Public Health 2016;16:1-7.

16 Kessler RC, Amminger GP, Aguilar-Gaxiolac S, et al. Age of onset of mental disorders: a review of recent literature. Curr Opin Psychiatry 2007;20:359-64.

17 Carter T, Morres ID, Meade O, et al. The effect of exercise on depressive symptoms in adolescents: a systematic review and metaanalysis. J Am Acad Child Adolesc Psychiatry 2016;55:580-90.

18 Bailey AP, Hetrick SE, Rosenbaum S, et al. Treating depression with physical activity in adolescents and young adults: a systematic review and meta-analysis of randomised controlled trials. Psychol Med 2018;48:1068-83.

19 Rodriguez-Ayllon M, Cadenas-Sánchez C, Estévez-López F, et al. Role of physical activity and sedentary behavior in the mental health of preschoolers, children and adolescents: a systematic review and meta-analysis. Sports Med 2019;49:1383-410. 
20 Caspersen CJ, Powell KE, Christenson GM. Physical activity, exercise, and physical fitness: definitions and distinctions for healthrelated research. Public Health Rep 1985;100:126-31.

21 Thompson PD, Arena R, Riebe D, et al. ACSM's New Preparticipation Health Screening Recommendations from ACSM's Guidelines for Exercise Testing and Prescription, Ninth Edition. Curr Sports Med Rep 2013;12:215-7.

22 Kelly P, Williamson C, Niven AG, et al. Walking on sunshine: Scoping review of the evidence for walking and mental health. Br J Sports Med 2018;52:800-6.

23 Australian Government. The Department of health. Australia's physical activity and sedentary behaviour guidelines and the Australian 24-hour movement guidelines Canberra, 2019. Available: https://www.health.gov.au/internet/main/publishing.nsf/Content/ health-pubhlth-strateg-phys-act-guidelines

24 Services. UDoHaH. Physical activity guidelines for Americans. 2nd edition. Washington, U.S.A: Department of Health and Human Services, 2018.

25 Bull FtEWG. Physical activity guidelines in the U.K. review and recommendations. Loughborough University, 2010.

26 Tricco AC, Lillie E, Zarin W, et al. PRISMA extension for scoping reviews (PRISMA-ScR): checklist and explanation. Ann Intern Med 2018;169:467-73

27 Arksey H, O'Malley L. Scoping studies: towards a methodological framework. Int J Soc Res Methodol 2005;8:19-32.

28 Hetrick SE, Parker AG, Callahan P, et al. Evidence mapping: illustrating an emerging methodology to improve evidence-based practice in youth mental health. J Eval Clin Pract 2010;16:1025-30.

29 American College of Sports Medicine Position Stand. American College of sports medicine position stand. The recommended quantity and quality of exercise for developing and maintaining cardiorespiratory and muscular fitness, and flexibility in healthy adults. Med Sci Sports Exerc 1998;30:975-91.

30 Norton K, Norton L, Sadgrove D. Position statement on physical activity and exercise intensity terminology. J Sci Med Sport 2010;13:496-502.

31 Pascoe MC, Bailey AP, Craike M, et al. Physical activity and exercise interventions for mental disorders in young people: a scoping review in clinical populations. The Lancet Psychiatry.

32 De Silva S, Bailey AP, Parker AG, et al. Open-Access evidence database of controlled trials and systematic reviews in youth mental health. Early Interv Psychiatry 2018;12:474-7.

33 Garber CE, Blissmer B, Deschenes MR, et al. American College of sports medicine position stand. quantity and quality of exercise for developing and maintaining cardiorespiratory, musculoskeletal, and neuromotor fitness in apparently healthy adults: guidance for prescribing exercise. Med Sci Sports Exerc 2011;43:1334-59.

34 Ainsworth BE, Haskell WL, Whitt MC, et al. Compendium of physical activities: an update of activity codes and Met intensities. Med Sci Sports Exerc 2000;32:S498-516.

35 MacMahon JR, Gross RT. Physical and psychological effects of aerobic exercise in delinquent adolescent males. Arch Pediatr Adolesc Med 1988;142:1361-6.

36 Balkin RS, Tietjen-Smith T, Caldwell C, et al. The utilization of exercise to decrease depressive symptoms in young adult women. Adultspan J 2007;6:30-5.

37 Hemat-Far A, Shahsavari A, Mousavi SR. Effects of selected aerobic exercises on the depression and concentrations of plasma serotonin in the depressed female students aged 18 to 25 . J Appl Res 2012;12:47-52.

38 Smith JA, Greer T, Sheets T, et al. Is there more to yoga than exercise? Altern Ther Health Med 2011;17:22-9.

39 Nabkasorn C, Miyai N, Sootmongkol A, et al. Effects of physical exercise on depression, neuroendocrine stress hormones and physiological fitness in adolescent females with depressive symptoms. Eur J Public Health 2006;16:179-84.

40 Bonhauser M, Fernandez G, Püschel K, et al. Improving physical fitness and emotional well-being in adolescents of low socioeconomic status in Chile: results of a school-based controlled trial. Health Promot Int 2005;20:113-22.

41 Melnyk BM, Jacobson D, Kelly S, et al. Improving the mental health, healthy lifestyle choices, and physical health of Hispanic adolescents: a randomized controlled pilot study. J Sch Health 2009;79:575-84.

42 Melnyk BM, Jacobson D, Kelly S, et al. Promoting healthy lifestyles in high school adolescents: a randomized controlled trial. Am J Prev Med 2013;45:407-15.

43 Noggle JJ, Steiner NJ, Minami T, et al. Benefits of yoga for psychosocial well-being in a US high school curriculum: a preliminary randomized controlled trial. J Dev Behav Pediatr 2012;33:193-201.
44 Khalsa SBS, Hickey-Schultz L, Cohen D, et al. Evaluation of the mental health benefits of yoga in a secondary school: a preliminary randomized controlled trial. J Behav Health Serv Res 2012;39:80-90.

45 Sabourin BC, Watt MC, Krigolson OE, et al. Two interventions decrease anxiety sensitivity among high anxiety sensitive women: could physical exercise be the key? J Cogn Psychother 2016;30:131-46.

46 Cecchini-Estrada J-A, Méndez-Giménez A, Cecchini C, et al. Exercise and Epstein's target for treatment of depressive symptoms: a randomized study. Int J Clin Health Psychol 2015;15:191-9.

47 Khorvash M, Askari A, Rafiemanzelat F, et al. An investigation on the effect of strength and endurance training on depression, anxiety, and C-reactive protein's inflammatory biomarker changes. J Res Med Sci 2012;17:1072-6.

48 Weinstock J, Capizzi J, Weber SM, et al. Exercise as an intervention for sedentary hazardous drinking college students: a pilot study. Ment Health Phys Act 2014;7:55-62.

49 Weinstock J, Petry NM, Pescatello LS, et al. Sedentary college student drinkers can start exercising and reduce drinking after intervention. Psychol Addict Behav 2016;30:791-801.

50 Roth DL, Holmes DS. Influence of aerobic exercise training and relaxation training on physical and psychologic health following stressful life events. Psychosom Med 1987;49:355-65.

51 Rotheram-Borus MJ, Tomlinson M, Durkin A, et al. Feasibility of using soccer and job training to prevent drug abuse and HIV. AIDS Behav 2016;20:1841-50.

52 Hilyer JCet al. Physical fitness training and counseling as treatment for youthful offenders. J Couns Psychol 1982;29:292-303.

53 O'Dougherty M, Hearst MO, Syed M, et al. Life events, perceived stress and depressive symptoms in a physical activity intervention with young adult women. Ment Health Phys Act 2012;5:148-54.

54 Robledo-Colonia AF, Sandoval-Restrepo N, Mosquera-Valderrama YF, et al. Aerobic exercise training during pregnancy reduces depressive symptoms in nulliparous women: a randomised trial. $J$ Physiother 2012;58:9-15.

55 Wipfli B, Landers D, Nagoshi C, et al. An examination of serotonin and psychological variables in the relationship between exercise and mental health. Scand J Med Sci Sports 2011;21:474-81.

56 Daley AJ, Copeland RJ, Wright NP, et al. Exercise therapy as a treatment for psychopathologic conditions in obese and morbidly obese adolescents: a randomized, controlled trial. Pediatrics 2006;118:2126-34

57 Gallego J, Aguilar-Parra JM, Cangas AJ, et al. Effect of a mindfulness program on stress, anxiety and depression in university students. Span J Psychol 2014;17:E109.

58 Taspinar B, Aslan UB, Agbuga B, et al. A comparison of the effects of hatha yoga and resistance exercise on mental health and wellbeing in sedentary adults: a pilot study. Complement Ther Med 2014;22:433-40.

59 Baghurst T, Kelley BC. An examination of stress in college students over the course of a semester. Health Promot Pract 2014;15:438-47.

60 Aras D, Ewert AW. The effects of eight weeks sport rock climbing training on anxiety. Acta Medica Mediterranea 2016;32:223-30.

61 Norris R, Carroll D, Cochrane R. The effects of physical activity and exercise training on psychological stress and well-being in an adolescent population. J Psychosom Res 1992;36:55-65.

62 Berger BG, Friedman E, Eaton M. Comparison of jogging, the relaxation response, and group interaction for stress reduction. $J$ Sport Exerc Psychol 1988;10:431-47.

63 Bao X, Jin K. The beneficial effect of tai chi on self-concept in adolescents. Int J Psychol 2015;50:101-5.

64 Ekkekakis P, Parfitt G, Petruzzello SJ. The Pleasure and displeasure people feel when they exercise at different intensities: decennial update and progress towards a tripartite rationale for exercise intensity prescription. Sports Med 2011;41:641-71.

65 Lee HH, Emerson JA, Williams DM. The exercise-affect-adherence pathway: an evolutionary perspective. Front Psychol 2016;07:1285.

66 Parker AG, Hetrick SE, Jorm AF, et al. The effectiveness of simple psychological and physical activity interventions for high prevalence mental health problems in young people: a factorial randomised controlled trial. J Affect Disord 2016;196:200-9.

67 Craft LL. Exercise and clinical depression: examining two psychological mechanisms. Psychol Sport Exerc 2005;6:151-71.

68 Carter T, Guo B, Turner D, et al. Preferred intensity exercise for adolescents receiving treatment for depression: a pragmatic randomised controlled trial. BMC Psychiatry 2015;15:247.

69 Craft LL, Perna FM, Freund KM, et al. Psychosocial correlates of exercise in women with self-reported depressive symptoms. J Phys Act Health 2008;5:469-80. 
70 Ryan RM, Deci EL. Self-determination theory and the facilitation of intrinsic motivation, social development, and well-being. Am Psychol 2000;55:68-78.

71 Wandersman A, Florin P. Community interventions and effective prevention. Am Psychol 2003;58:441-8.

72 Ennett ST, Ringwalt CL, Thorne J, et al. A comparison of current practice in school-based substance use prevention programs with meta-analysis findings. Prev Sci 2003;4:1-14.

73 Mendelson T, Tandon SD. Prevention of depression in childhood and adolescence. Child Adolesc Psychiatr Clin N Am 2016;25:201-18.

74 Romano JL. Psychoeducational interventions for stress management and well-being. J Couns Dev 1992;71:199-202.

75 Berger BG, Friedman E. Comparison of jogging, the relaxation response, and group interaction for stress reduction. J Sport Exerc Psychol 1988;10:431-47.

76 Deci E, Ryan RM. Handbook of Self-Determination research. Rochester, NY: Univ. Rochester Press, 2002.

77 Lubans D, Richards J, Hillman C, et al. Physical activity for cognitive and mental health in youth: a systematic review of mechanisms. Pediatrics 2016;138:e20161642.
78 Ryff CD, Keyes CLM. The structure of psychological well-being revisited. J Pers Soc Psychol 1995;69:719-27.

79 Lederman O, Suetani S, Stanton R, et al. Embedding exercise interventions as routine mental health care: implementation strategies in residential, inpatient and community settings. Australas Psychiatry 2017;25:451-5.

80 Craike MJ, Mosely K, Browne JL, et al. Associations between physical activity and depressive symptoms by weight status among adults with type 2 diabetes: results from diabetes MILES-Australia. $J$ Phys Act Health 2017;14:195-202.

81 Vallance JK, Winkler EAH, Gardiner PA, et al. Associations of objectively-assessed physical activity and sedentary time with depression: NHANES (2005-2006). Prev Med 2011;53:284-8

82 Brunes A, Augestad LB, Gudmundsdottir SL. Personality, physical activity, and symptoms of anxiety and depression: the HUNT study. Soc Psychiatry Psychiatr Epidemiol 2013;48:745-56.

83 Berger BG, Owen DR. Stress reduction and mood enhancement in four exercise modes: swimming, body conditioning, hatha yoga, and fencing. Res Q Exerc Sport 1988;59:148-59. 